Research Article

\title{
The New Plastic Flow Machining Process for Producing Thin Sheets
}

\author{
Viet Q. Vu, ${ }^{1,2}$ Yan Beygelzimer, ${ }^{2,3}$ Roman Kulagin, ${ }^{4}$ and Laszlo S. Toth $\mathbb{D}^{1,2}$ \\ ${ }^{1}$ Université de Lorraine, CNRS, Arts et Métiers ParisTech, LEM3, F-57000 Metz, France \\ ${ }^{2}$ Laboratory of Excellence on Design of Alloy Metals for Low-Mass Structures (DAMAS), Université de Lorraine, Metz, France \\ ${ }^{3}$ Donetsk Institute for Physics and Engineering Named After O.O. Galkin, National Academy of Sciences of Ukraine, \\ Kyiv, Ukraine \\ ${ }^{4}$ Institute of Nanotechnology (INT), Karlsruhe Institute of Technology (KIT), Karlsruhe, Germany
}

Correspondence should be addressed to Laszlo S. Toth; laszlo.toth@univ-lorraine.fr

Received 28 April 2018; Accepted 12 July 2018; Published 16 September 2018

Academic Editor: Pavel Lejcek

Copyright (c) 2018 Viet Q. Vu et al. This is an open access article distributed under the Creative Commons Attribution License, which permits unrestricted use, distribution, and reproduction in any medium, provided the original work is properly cited.

\begin{abstract}
A new severe plastic deformation (SPD) process called plastic flow machining (PFM) was recently proposed to produce thin sheets with gradient structures. In the present paper, the role of the die geometry is investigated by studying the effects of the produced sheet thickness $(h)$ on the material properties of commercial pure Aluminum (Al1050) processed by PFM. The obtained experimental results show that an increase of $h$ in the range of 0.65 to $1.5 \mathrm{~mm}$ improved the formation efficiency of the sheet. Microstructures of the produced sheets show gradient structures with an average grain size varying from 0.8 to $3.81 \mu \mathrm{m}$ across the sheet thickness. Both experiments and finite element (FE) simulations document that the degree of the gradient in the microstructure became more significant when $h$ was increased. Sheets produced by PFM exhibited a better strength-ductility balance than sheets obtained in other SPD processes. Tensile strength of 160-175 MPa and total ductility of $18-25 \%$ were obtained for the processed samples after PFM. A rise of $h$ from 0.65 to $1.5 \mathrm{~mm}$ lowered the strength but enhanced the ductility of the produced sheet, which is due to the coarser microstructure at larger values of $h$.
\end{abstract}

\section{Introduction}

Severe plastic deformation (SPD) has been acclaimed as an effective technique for producing metals with superior properties which are unattainable by conventional thermomechanical processing. To date there have been a great number of SPD processes proposed, such as high pressure torsion (HPT) [1], equal channel angular pressing (ECAP) [2], accumulative roll bonding (ARB) [3], twist extrusion (TE) [4], repetitive corrugation and straightening (RCS) [5], to name a few. One of the most prominent benefits of SPD is its ability to transform an initial coarse-grained (CG) structure into an ultrafine-grained (UFG) structure at room temperature, which significantly increases the mechanical strength of the processed material via grain-boundary strengthening (also known as conventional Hall-Petch strengthening $[6,7])$. The main drawback of SPD is that the considerable increase of strength is inevitably accompanied by a significant loss of ductility which might limit the employment of this technique in industrial applications [8-11]. Therefore, seeking a method imparting high strength while maintaining reasonable ductility is an enormous challenge in SPD research. A possible solution to this issue is to develop SPD methods which are able to produce metals with appropriate microstructures. A novel SPD process was recently proposed-called plastic flow machining (PFM) - which can produce gradient structures $[12,13]$. In PFM, a surface layer of a bulk workpiece is separated via a controllable lateral extrusion process to transform it into a fin or sheet with an ultrafine-grained and gradient structure, in a single deformation step, which is basically simple shear. PFM has a similar feature with another recently invented process, called large strain extrusion machining (LSEM) $[14,15]$ in the sense that in LSEM continuous metal fins with UFG 
structure can also be produced by separating a surface layer from a bulk sample. The difference is that in LSEM a cutting tool is used together with a constraining channel, while in PFM, an extrusion process produces the fin. However, the microstructure produced by PFM is heterogeneous while it is mostly homogeneous in LSEM, and the hydrostatic pressure produced in the deformation zone in PFM is likely to be higher than that in LSEM. PFM also exhibits a common feature with nonequal channel angular pressing (NECAP) $[16,17]$ in terms of the working principle. Indeed, lateral extrusion in PFM works in the same way as in NECAP. However, the main difference is that in PFM, only the surface layer of the sample is deformed while the whole bulk sample is deformed in NECAP. This allows PFM to reduce the load required for processing.

PFM was successfully applied on commercial pure Aluminum 1050 to fabricate fins with different thicknesses. The obtained fins, produced through the lateral extrusion process, exhibited gradients in terms of plastic strain, microstructure, and texture across their thickness. The mechanical properties and formability of those fins were found to be excellent. For example, a $0.65 \mathrm{~mm}$ thickness fin, produced by the one-step PFM operation, shows tensile strength equivalent to that obtained from four passes of incremental equal channel angular pressing (I-ECAP). The uniform elongation is also up to four times higher than that received after four I-ECAP passes [13]. The average Lankford value attained from the tensile tests at different directions of this fin is 0.92 , which is much higher than that obtained from conventional rolling which ranges from 0.5 to 0.85 [13]. Owning to the ability to fabricate metallic sheets with superior mechanical properties and formability, PFM shows great potentials for industrial applications, and it is patented worldwide [12]. The aim of this paper is to give more information on this new process, especially concerning the role of the die geometry.

\section{Experimental and Simulation Procedures}

2.1. Experimental Procedures. Commercial pure Al1050 samples were processed by PFM at room temperature. A description of the process was presented in a previous work [13] and schematically presented in Figure 1. Fins were produced with different thicknesses up to $1.5 \mathrm{~mm}$, while keeping other geometrical parameters constant: the die angle $\alpha=120^{\circ}$ and the heights of the two die channels: $H_{0}=20 \mathrm{~mm}$ and $H_{1}=18 \mathrm{~mm}$. A thickness ratio parameter was introduced for the fin, which is the ratio between the thickness of the fin and the thickness of the layer removed from the bulk:

$$
t=\frac{h}{\left(H_{0}-H_{1}\right)} .
$$

The effects of this parameter on the fin formation and its microstructure and mechanical properties were investigated. As the die was constantly the same with $H_{0}-H_{1}=2 \mathrm{~mm}$, for the results presented in this work, $t$ and $h$ are simply related by $t=h / 2$. The experiments were conducted applying a back pressure of $110 \mathrm{MPa}$ and also without back pressure.
The microstructures across the thickness of the fins were characterized by scanning electron microscopy (SEM) and electron back-scattering diffraction (EBSD) operated in a JEOL JSM-6500F field-emission gun-scanning electron microscope. Data acquisition and analyses were carried out by the Aztec HKL and the ATOM software [18], respectively. For the detection of grain boundaries, disorientation angles between neighboring pixels were required to be greater than $5^{\circ}$. In order to obtain and compare mechanical properties of the fins produced at different gap widths, tensile tests were performed at room temperature. The tensile direction was set parallel to the longitudinal direction of the fin, and the strain rate was $0.001 \mathrm{~s}^{-1}$.

2.2. Simulation Procedures. The commercial DEFORM2D/3D V11.0 FE code was used for the FE simulations to obtain insight into the stress and strain distributions within the workpiece during the PFM process. All parts of deforming tools were defined as rigid bodies, whereas the workpiece was represented by 10000 tetragonal deformable elements. Adaptive meshing was used to accommodate large strains during the material flow within the lateral channel. The material behavior was taken isotropic using the von Mises model with the hardening law $\sigma=180 \varepsilon_{\mathrm{vM}}^{0.23}(\mathrm{MPa})$ ( $\sigma$ is the von Mises equivalent stress, and $\varepsilon_{\mathrm{vM}}$ is von Mises strain). The parameters of the hardening law were obtained by approximating the stress-strain curve of Al1050 from [19]. Friction was modeled by the Siebel friction law $\tau=0.2 \sigma$.

\section{Results and Discussion}

3.1. The Effect of Die Geometry and Back Pressure on the Formation of the Fin. A special feature of PFM is that both forward and lateral extrusions occur concurrently during processing; see the illustration in Figure 1(b). The sample has a cross section of $H_{0} W$ which changes into $H_{1} W$ after processing, where $W$ is the width of the workpiece. The outgoing fin has a cross section of $h D$. During the process, the incoming flow $Q_{0}$ is bifurcating into two flows: $Q_{1}$ and $Q_{2}$. These three flows are defined in terms of volumes moving across the cross sections per unit time:

$$
\text { incoming flow: } Q_{0}=U_{0} H_{0} W \text {, }
$$

forward extrusion flow: $Q_{1}=U_{1} H_{1} W$,

$$
\text { lateral extrusion flow: } Q_{2}=U_{2} h W \text {, }
$$

where $U_{0}, U_{1}$, and $U_{2}$ are the respective material flow velocities. Now, we introduce a parameter $x$ which is the lateral extrusion ratio defined by the fraction of the metal that flows into the lateral slit:

$$
x=\frac{Q_{2}}{Q_{0}} .
$$

Using Equations (2) and (4), we obtain 


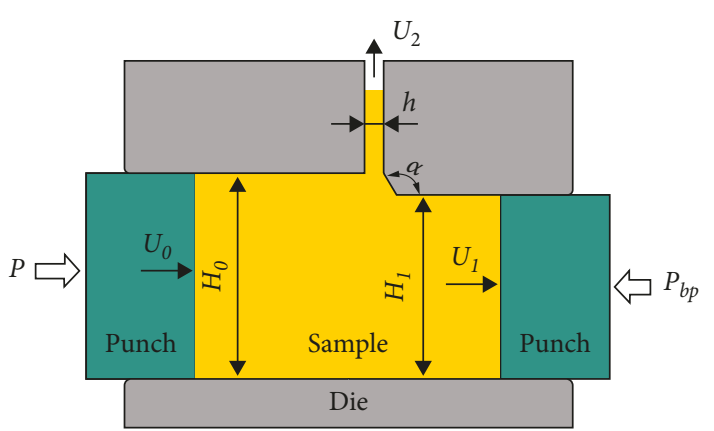

(a)

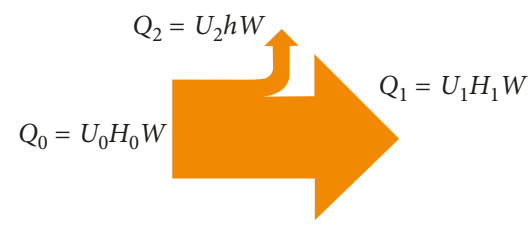

(b)

FIgURE 1: Schematic illustration of the PFM process: (a) die geometry and (b) metal flow. $P$ and $P_{\mathrm{bp}}$ are the pressing pressure and back pressure, respectively. $H_{0}, H_{1}, h$, and $\alpha$ are the geometrical dimensions. $W$ is the width dimension. $U_{0}, U_{1}$, and $U_{2}$ are the velocities, and $Q_{0}, Q_{1}$, and $Q_{2}$ are the volumes per unit time of the metal flows.

$$
x=\frac{U_{2} h}{U_{0} H_{0}},
$$

where $U_{2}$ and $U_{0}$ velocities can be expressed in terms of time $T$ of the extrusion, the length of the fin $l$, and the displacement of the sample $L_{0}$ in contact with the pressing punch:

$$
\begin{aligned}
& U_{2}=\frac{l}{T}, \\
& U_{0}=\frac{L_{0}}{T},
\end{aligned}
$$

which leads to

$$
x=\frac{l h}{L_{0} H_{0}} .
$$

As can be seen from this expression, the parameter $x$ is fully defined by simple geometrical parameters that can be readily measured, just like the parameter $t$ from Equation (1). The maximum possible value of $x$ is 1 ; it happens when the back-pressure punch does not move: it is fixed. This situation corresponds to the case of nonequal channel angular pressing (NECAP) which was examined analytically and experimentally in $[16,17,20]$.

The lateral extrusion ratio $(x)$ is plotted as a function of the fin thickness ratio in Figure 2, from a number of experiments conducted with and without back pressure.

As can be seen, the lateral extrusion ratio increases with the gap-width size, and it is more prominent when processing with back pressure. Therefore, in order to enhance the formation of the fin and improve efficiency, PFM needs to be carried out with sufficiently high fin thickness
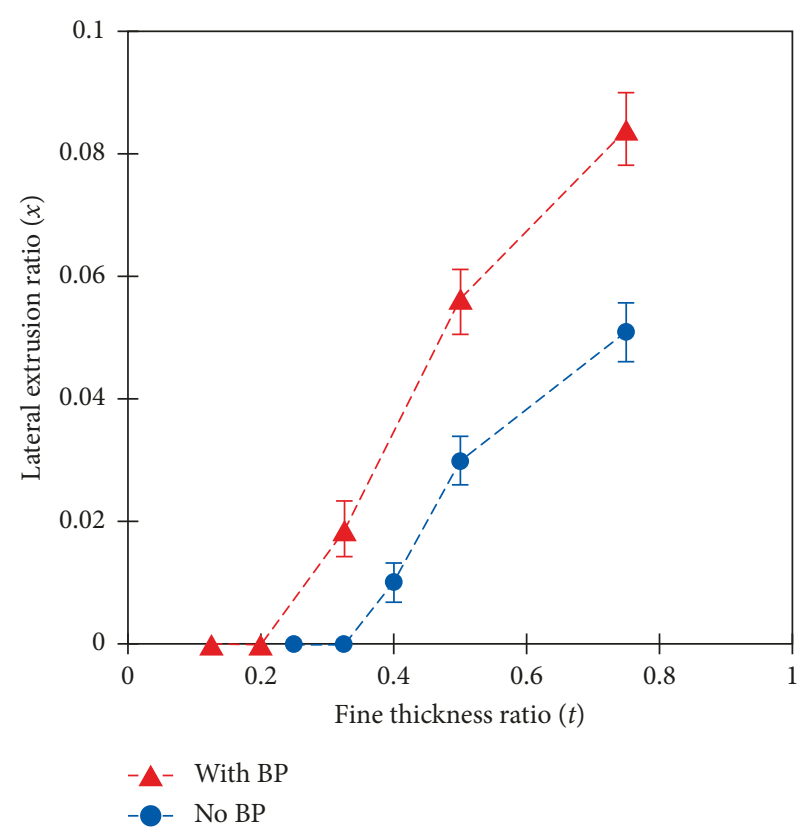

Figure 2: Measured dependence of the lateral extrusion ratio on the fin thickness ratio, under a back pressure of $110 \mathrm{MPa}$ and also without back pressure.

ratio and with the assistance of a back pressure. When $t$ was smaller than 0.35 , the fin was not produced in the absence of back pressure. With back pressure, this critical value was reduced to 0.2 . This means that at small-gap widths $(h)$, the lateral extrusion stops at a certain point, and the forward extrusion is predominant during the process. High friction and the formation of a dead metal zone near the entrance of the lateral slit might be the causes that obstructed the lateral flow at narrow-gap widths of the side channel. With the rise of $t$, the process was improved. Bearing all the above in mind, when designing a PFM die, for a given fin thickness, one should choose a reasonable value of $t$.

3.2. The Effect of Fin Thickness Ratio on Strain and Microstructure in the Fin. The microstructures of three fins corresponding to three-fin thickness ratios, $0.325,0.5$, and 0.75 , were characterized by EBSD measurements. The case of $t=$ 0.75 was selected to show the microstructure of the fin, see Figure 3(b). Clearly, grain refinement with a microstructure gradient across the thickness can be seen.

A significant grain refinement and the strain gradient across the fin thickness are documented in Figure 4. The initial grain size of about $100 \mu \mathrm{m}$ was refined to 3.81, 2.1, and $0.85 \mu \mathrm{m}$ in the left edge area (LEA), middle area (MA), and right edge area (REA), respectively, of the fin.

Regarding the strain gradient across the fin thickness, the reason for this is that material points flow along different strain paths while moving from the bulk workpiece into the fin. Each time the material point alters its flow direction, a shear strain is adding and accumulating. The diversity of 


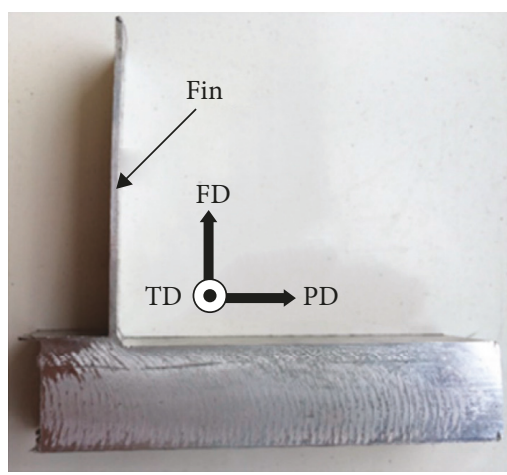

(a)

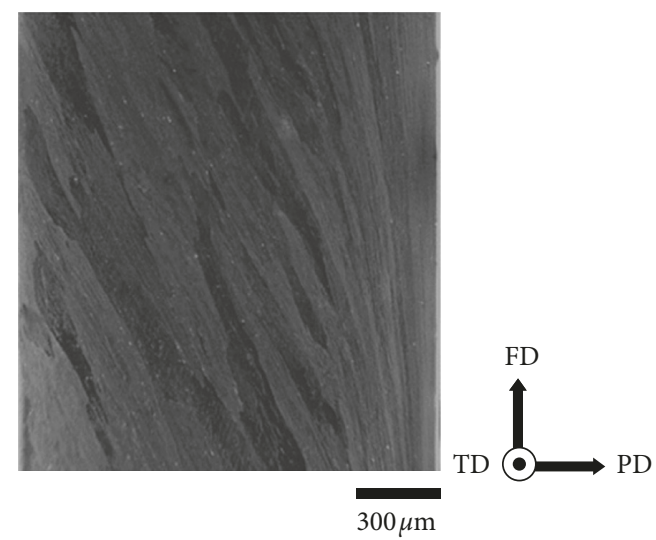

(b)

Figure 3: (a) A sample after PFM processing. (b) SEM microstructure across the thickness of the fin for the case of $t=0.75$.

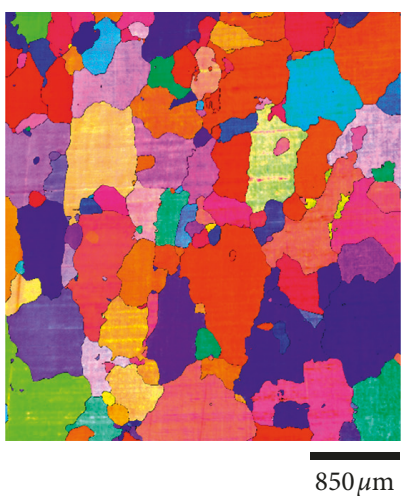

(a)

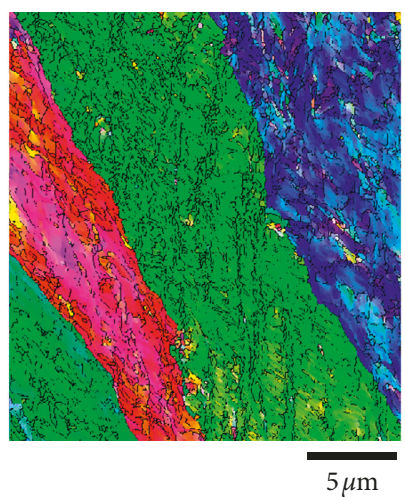

(b)

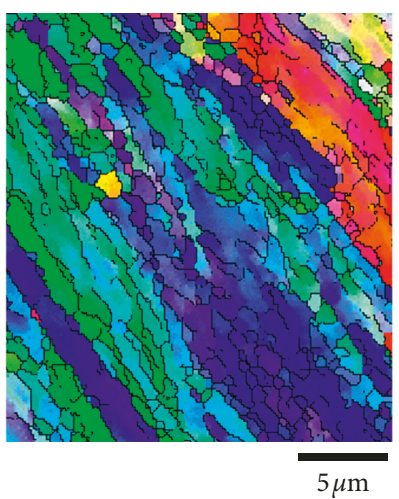

(c)

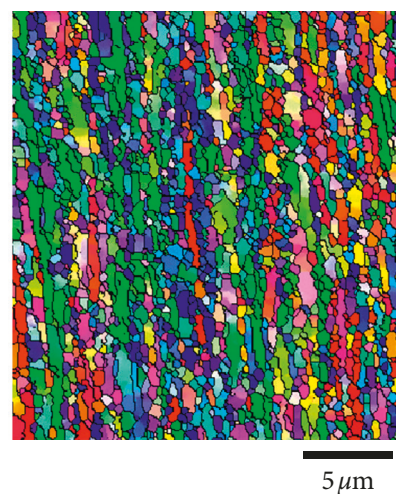

(d)
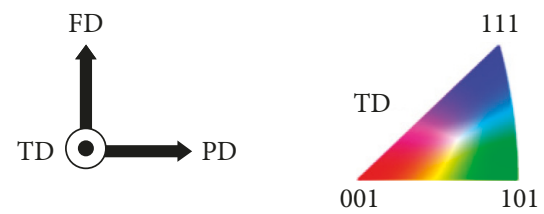

Figure 4: EBSD microstructures of (a) initial sample and (b) left edge area (LEA), (c) middle area (MA), and (d) right edge area (REA), respectively, of the fin for the case of $t=0.75$.

strain paths forms a complex strain field in the deformation zone situated at the intersection of the forward and lateral channels. High friction at the interface between the right side of the fin and the $120^{\circ}$ inclined edge of the die (Figure 1(a)) also contributes to this strain gradient.

The grain sizes corresponding to three different fin thickness ratios can be evaluated using Figure 5. As can be seen, the grain size increased almost linearly across the fin thickness, from the REA to the LEA in all three cases of the fin thickness ratios. In addition, with the increase of $t$ from 0.325 to 0.75 , the average grain size in the REA did not change considerably, from 0.8 to $0.85 \mu \mathrm{m}$, respectively. At the same time, the average grain sizes in the MA and LEA were significantly increased, from 1.4 to $2.1 \mu \mathrm{m}$ and from 1.9 to $3.81 \mu \mathrm{m}$, respectively. It is widely accepted in SPD research that the significant microstructure refinement under large plastic strain is associated with a continuous dynamic recrystallization phenomenon. This process involves grain subdivision caused by the buildup of GNDs and new fine grain formation with high-angle grain boundaries (HAGBs) during large deformation at room temperature $[21,22]$. The smaller grain size change in the REA compared with that in the MA and LEA when $t$ is varied implies that the strain imposed in the REA was less dependent on the geometry of the die. This is because the strain in the REA resulted not only from the abrupt change of material point trajectory (which depends on the die geometry) but also from the effect of the high friction at the interface between the right side of the fin and the die surface. An average gradient in grain size $(g)$ can be defined as the slope of the best-fit line which represents the dependence of grain size on the distance (Figure 5). This gradient was found to be the same $2.1 \times 10^{-3}$ for all $t$ values from 0.0325 to 0.75 . 


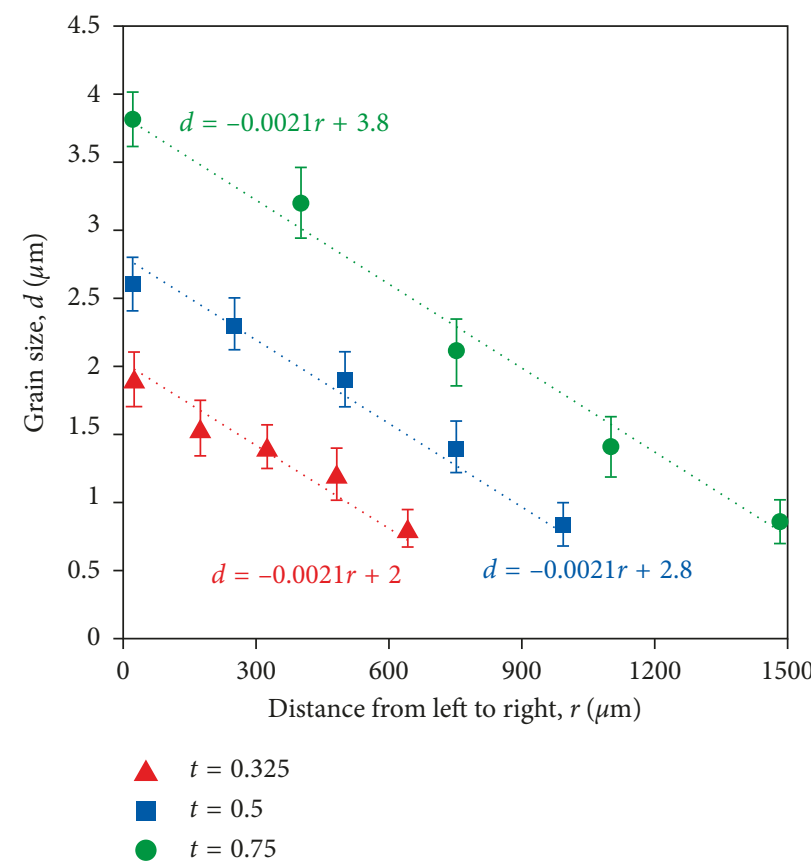

FIGURE 5: Variation of grain size within the fin for three values of the fin thickness ratio $t$.

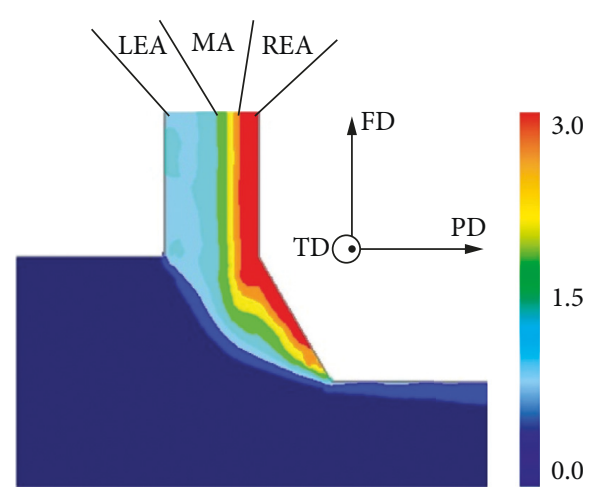

Effective strain $(\mathrm{mm} / \mathrm{mm})$

(a)

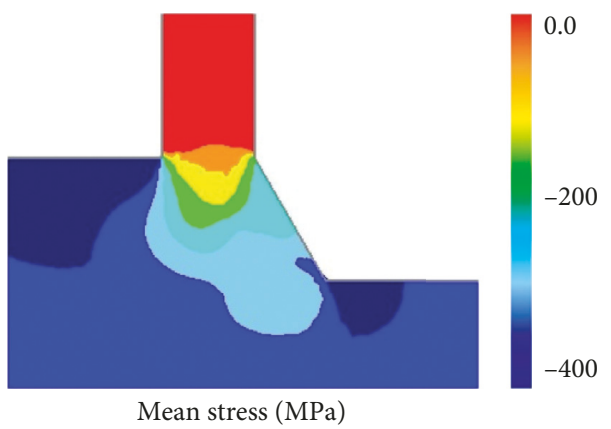

(c)

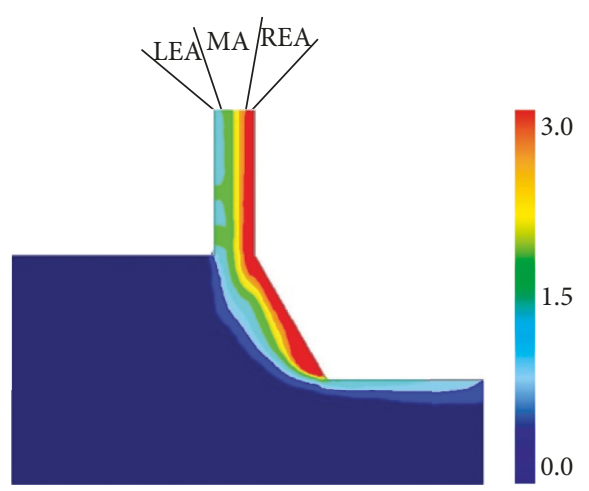

Effective strain $(\mathrm{mm} / \mathrm{mm})$

(b)

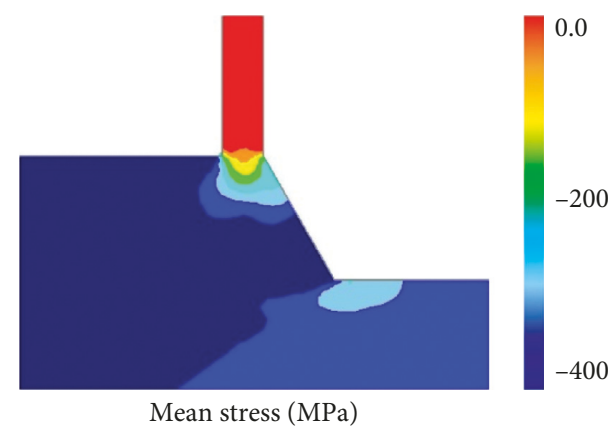

(d)

Figure 6: Results of FE simulations on strain and stress distributions for (a) and (c) $t=0.75$ and for (b) and (d) $t=0.325$. The maximum strain is 4 for both cases. LEA, MA, and REA are the left edge area, middle area, and right edge areas, respectively.

The results of the FE simulations obtained for strain and stress distributions for the two values of $t(0.75$ and 0.325$)$ are displayed in Figure 6. As can be seen from Figures 6(a) and 6(b), the top layer of the bulk sample is heavily deformed within the deformation zone adjacent to the entry of the lateral channel. The heterogeneous deformation in 
this zone leads to the strain gradient of the produced fin, in which the strain values increase across the fin thickness from the left to the right. One can analyze this strain gradient by dividing the fin into three strain areas, namely, the left edge area (LEA), the middle area (MA), and the right edge area (REA). The greater deformation in the HSA can be attributed to high friction generated at the contact between the material and the inclined edge of the die, under high compression pressure. The REAs in both cases $t=$ 0.75 and 0.325 exhibit a similar range of von Mises strain values from 3 to 4 . However, the LEAs show different features in the two cases. In the case $t=0.75$ (Figure 6(a)), the REA displays lower von Mises strain values (from 1 to 1.5 ) than in the case $t=0.325$ (about 1.5). This is consistent with the experimental results that the average grain size in the LEA decreased from 3.8 to 1.9 when $t$ increased from 0.75 to 0.325 , but that in the REA did not change considerably (from 0.85 to $0.8 \mu \mathrm{m}$ ). Figures 6(b) and 6(d) show the stress distribution for the two different $t$ values. In both cases, high compressive stresses (with a maximum value of $400 \mathrm{MPa}$ ) are generated in the deformation zone due to a large hydraulic pressure which is enhanced by the applied back pressure. Therefore, microfractures such as microcracks and voids are suppressed in PFM, improving in this way the workability of the fin.

3.3. Mechanical Properties and the Effect of the Fin Thickness Ratio. The tensile test results of the initial and PFMprocessed samples corresponding to different fin thickness ratios, ranging from 0.325 to 0.75 , are presented in Figure 7 (a). The geometry of tested samples is displayed in Figure 7(b) with $h$ being the thickness of the sample. As can be seen, a significant increase in strength, about more than 2.5 times, was achieved in all produced fins. Good elongations-which were far higher than those in other SPD counterparts-were also attained. A comparison of ductility obtained at a similar strength range for Al1050 from several SPD processes is presented in Table 1 . The data were collected from different studies. As can be seen, in a comparable range of UTS strength, PFM provides higher uniform and total elongations than other SPD processes. For example, compared with eight ECAP passes route $\mathrm{Bc}$ at room temperature (RT) followed by annealing at varying conditions, one PFM pass shows about twice higher uniform elongation and a 1.5 times higher total elongation. Another example is that when comparing with seven ARB passes at RT, one PFM pass presents about four to five times higher uniform elongation. This again highlights the novelty of PFM in terms of imparting high strength while maintaining reasonable ductility and sheds light on the benefit of the gradient microstructure in improving the strengthductility relation.

Regarding the impact of the fin thickness ratio on the mechanical properties, the strength decreased with the increase of $t$. A decrease from nearly 180 to $160 \mathrm{MPa}$ in UTS took place when $t$ varied from 0.325 to 0.75 . The opposite trend was seen for the ductility: the uniform elongation increased from 6 to $8 \%$, and the total elongation increased from 18 to $25 \%$. These results can be explained by correlating

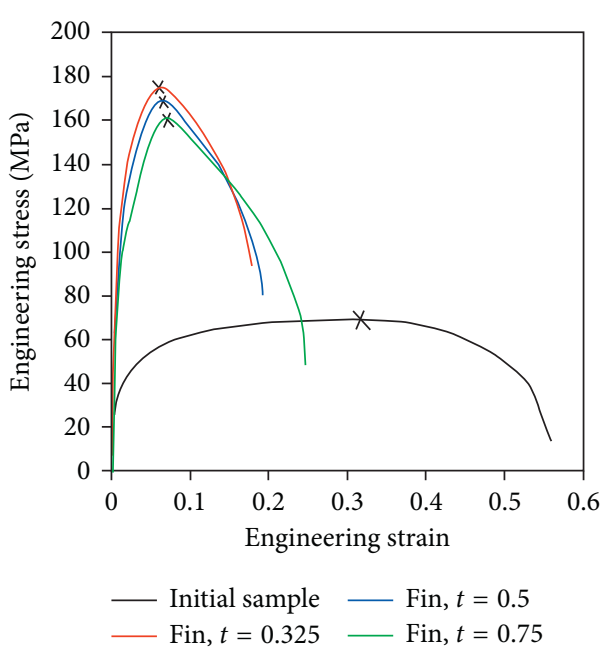

(a)

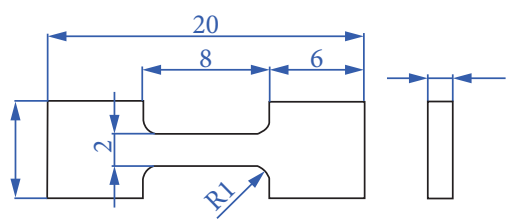

(b)

FIGURE 7: (a) Engineering stress-strain curves of the initial sample and the fins for varying thickness ratios. The superimposed crosses show the end of uniform elongation. (b) The geometry of the tensile specimens.

them with the effect of the fin thickness ratio on the strain and microstructure that are presented in the previous sections. The grain sizes in all areas, the LEA, MA, and REA, increased with the increase of $t$ (Figure 5). This means that the microstructure generally became coarser when $t$ was increased, resulting in a decrease in strength and increase in ductility, as expected.

To this end, the mechanical properties of PFM-processed fins can be controlled by the fin thickness ratio. The target of obtaining high strength can be fulfilled by choosing a small $t$ value. In this case, good ductility, higher than that in other SPD techniques (Table 1), can be reached.

\section{Conclusions}

(i) Lateral and forward extrusions occur simultaneously during the PFM process. The process should be conducted with a back pressure to improve its efficiency. In the condition of processing with a back pressure of $110 \mathrm{MPa}$, when $t$ was smaller than 0.325 , the fin was not formed because the lateral extrusion stopped at a certain point and the forward extrusion became predominant during the process. With the rise of $t$ from 0.325 to 0.75 , the lateral extrusion ratio increased, improving the formation efficiency of the fin. 
TABLE 1: Tensile test results on Al1050 preprocessed by different SPD techniques.

\begin{tabular}{|c|c|c|c|c|c|c|c|}
\hline \multirow{2}{*}{ SPD technique } & \multicolumn{2}{|c|}{ UTS (MPa) } & \multicolumn{2}{|c|}{ Uniform El (\%) } & \multicolumn{2}{|c|}{ Total El (\%) } & \multirow{2}{*}{ Ref. } \\
\hline & Initial & Final & Initial & Final & Initial & Final & \\
\hline \multicolumn{8}{|l|}{1 pass $\mathrm{PFM}$ at $\mathrm{RT}$} \\
\hline$t=0.325$ & & $175 \pm 3$ & & $6 \pm 0.5$ & & $18 \pm 0.8$ & \\
\hline$t=0.5$ & $70 \pm 2$ & $169 \pm 3$ & $31 \pm 1$ & $7 \pm 0.5$ & $56 \pm 1$ & $20 \pm 0.8$ & This study \\
\hline$t=0.75$ & & $160 \pm 3$ & & $8 \pm 0.5$ & & $25 \pm 0.8$ & \\
\hline $\begin{array}{l}8 \text { ECAP passes route } \mathrm{Bc} \text { at } \mathrm{RT}+\text { annealing at varying } \\
\text { conditions }\end{array}$ & - & $140 / 175$ & - & $3 / 3.5$ & - & $10 / 15$ & {$[23]$} \\
\hline 4 incremental ECAP passes at RT & 76 & 177 & 35 & 1.5 & 55 & 15 & {$[24]$} \\
\hline $60 \%$ reduction in ASR at $\mathrm{RT}$ & 100 & 176 & 2 & 2 & 17 & 6.5 & {$[25]$} \\
\hline 7 ARB passes at RT & 110 & 150 & - & - & 12 & 9 & {$[26]$} \\
\hline 4 ECFE passes at RT & 78 & 122 & 32 & 1.5 & 43 & 14.7 & {$[27]$} \\
\hline
\end{tabular}

RT: room temperature; UTS: ultimate strength; El: elongation; Ref.: reference; ASR: asymmetric rolling; ARB: accumulative roll bonding; ECFE: equal channel forward extrusion.

(ii) Ultrafine-grained microstructure and gradient microstructure were obtained after one-step PFM deformation. The average grain size varied from 0.8 to $3.81 \mu \mathrm{m}$ across the fin thickness. When $t$ was increased from 0.325 to 0.75 , the degree of the gradient in the microstructure became more significant. This was in good agreement with the FEM simulation in which the strain gradient showed the same dependence on $t$.

(iii) PFM exhibits superior strength-ductility balance with respect to other SPD counterparts.

A tensile strength of $160-175 \mathrm{MPa}$ and total ductility of 18-25\% were obtained for Al1050 samples after one-step deformation. An increase of $t$ from 0.325 to 0.75 reduces the strength but improves the ductility of the produced fin. This can be attributed to the coarser microstructure obtained for larger $t$ values.

\section{Data Availability}

The data used to support the findings of this study are available from the corresponding author upon request.

\section{Conflicts of Interest}

The authors declare that there are no conflicts of interest regarding this publication.

\section{Acknowledgments}

This work was supported by the French State through the program "Investment in the future" operated by the $\mathrm{Na}$ tional Research Agency (ANR) and referenced by ANR-11LABX-0008-01 (Labex DAMAS). Viet Q. VU acknowledges the PhD scholarship awarded by the Vietnamese Government (Project 911).

\section{References}

[1] A. P. Zhilyaev and T. G. Langdon, "Using high-pressure torsion for metal processing: fundamentals and applications," Progress in Materials Science, vol. 53, no. 6, pp. 893-979, 2008.
[2] R. Z. Valiev and T. G. Langdon, "Principles of equal-channel angular pressing as a processing tool for grain refinement," Progress in Materials Science, vol. 51, no. 7, pp. 881-981, 2006.

[3] Y. Saito, H. Utsunomiya, N. Tsuji, and T. Sakai, "Novel ultrahigh straining process for bulk materials-development of the accumulative roll-bonding (ARB) process," Acta Materialia, vol. 47, no. 2, pp. 579-583, 1999.

[4] Y. Beygelzimer, V. Varyukhin, S. Synkov, and D. Orlov, "Useful properties of twist extrusion," Materials Science and Engineering: A, vol. 503, no. 1, pp. 14-17, 2009.

[5] Y. T. Zhu, H. Jiang, J. Huang, and T. C. Lowe, "A new route to bulk nanostructured metals," Metallurgical and Materials Transactions A, vol. 32, no. 6, pp. 1559-1562, 2001.

[6] E. O. Hall, "The deformation and ageing of mild steel: III discussion of results," Proceedings of the Physical Society. Section B, vol. 64, no. 9, pp. 747-753, 1951.

[7] N. J. Petch, "The cleavage strength of polycrystals," Journal of the Iron and Steel Institute, vol. 174, pp. 25-28, 1953.

[8] C. C. Koch, D. G. Morris, K. Lu, and A. Inoue, "Ductility of nanostructured materials," MRS Bulletin, vol. 24, no. 2, pp. 54-58, 1999.

[9] E. Ma, "Eight routes to improve the tensile ductility of bulk nanostructured metals and alloys," JOM, vol. 58, no. 4, pp. 49-53, 2006.

[10] N. Tsuji, Y. Ito, Y. Saito, and Y. Minamino, "Strength and ductility of ultrafine grained aluminum and iron produced by ARB and annealing," Scripta Materialia, vol. 47, no. 12, pp. 893-899, 2002.

[11] R. Valiev, "Nanostructuring of metals by severe plastic deformation for advanced properties," Nature Materials, vol. 3, no. 8, pp. 511-516, 2004.

[12] Y. Beygelzimer, L. S. Toth, and J.-J. Fundenberger, Procédé de Formation d'un Objet Plat Métallique à Grains Ultrafins, Patent: WO2017017341, Universite de Lorraine, Metz, France, 2015.

[13] V. Q. Vu, Y. Beygelzimer, L. S. Toth, J.-J. Fundenberger, R. Kulagin, and C. Chen, "The plastic flow machining: a new SPD process for producing metal sheets with gradient structures," Materials Characterization, vol. 138, pp. 208-214, 2018.

[14] M. Efe, W. Moscoso, K. P. Trumble, W. Dale Compton, and S. Chandrasekar, "Mechanics of large strain extrusion machining and application to deformation processing of magnesium alloys," Acta Materialia, vol. 60, no. 5, pp. 2031-2042, 2012. 
[15] W. Moscoso, M. R. Shankar, J. B. Mann, W. D. Compton, and S. Chandrasekar, "Bulk nanostructured materials by large strain extrusion machining," Journal of Materials Research, vol. 22, no. 1, pp. 201-205, 2011.

[16] A. Hasani, L. S. Tóth, and B. Beausir, "Principles of non-equal channel angular pressing," Journal of Engineering Materials and Technology, vol. 132, no. 3, article 031001, 2010.

[17] L. S. Tóth, R. Lapovok, A. Hasani, and C. Gu, "Non-equal channel angular pressing of aluminum alloy," Scripta Materialia, vol. 61, no. 12, pp. 1121-1124, 2009.

[18] B. Beausir and J.-J. Fundenberger, ATOM, Analysis Tool for Orientation Maps, Université de Lorraine, Metz, France, 2015, http://atom-software.eu/.

[19] M. Arzaghi, J. J. Fundenberger, L. S. Toth et al., "Microstructure, texture and mechanical properties of aluminum processed by high-pressure tube twisting," Acta Materialia, vol. 60, no. 11, pp. 4393-4408, 2012.

[20] C. F. Gu and L. S. Tóth, "Texture development and grain refinement in non-equal-channel angular-pressed Al"," Scripta Materialia, vol. 67, no. 1, pp. 33-36, 2012.

[21] R. Pippan, S. Scheriau, A. Taylor, M. Hafok, A. Hohenwarter, and A. Bachmaier, "Saturation of fragmentation during severe plastic deformation," Annual Review of Materials Research, vol. 40, no. 1, pp. 319-343, 2010.

[22] L. S. Toth and C. Gu, "Ultrafine-grain metals by severe plastic deformation," Materials Characterization, vol. 92, pp. 1-14, 2014.

[23] C. Y. Yu, P. W. Kao, and C. P. Chang, "Transition of tensile deformation behaviors in ultrafine-grained aluminum," Acta Materialia, vol. 53, no. 15, pp. 4019-4028, 2005.

[24] M. Salamati, S. Tamimi, S. Moturu, G. Sivaswamy, M. Qarni, and A. Rosochowski, "Microstructure and mechanical properties of Al-1050 during incremental ECAP," IOP Conference Series: Materials Science and Engineering, vol. 194, no. 1, article 012009, 2017.

[25] J. Jiang, Y. Ding, F. Zuo, and A. Shan, "Mechanical properties and microstructures of ultrafine-grained pure aluminum by asymmetric rolling," Scripta Materialia, vol. 60, no. 10, pp. 905-908, 2009.

[26] Y.-S. Kim, S. H. Kang, and D. H. Shin, "Effect of rolling direction on the microstructure and mechanical properties of accumulative roll bonding (ARB) processed commercially pure 1050 aluminum alloy," Materials Science Forum, vol. 503-504, pp. 681-686, 2006.

[27] M. Ebrahimi, F. Djavanroodi, S. Tiji, H. Gholipour, and C. Gode, "Experimental investigation of the equal channel forward extrusion process," Metals, vol. 5, no. 1, pp. 471-483, 2015. 


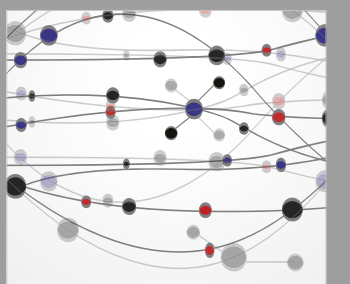

The Scientific World Journal
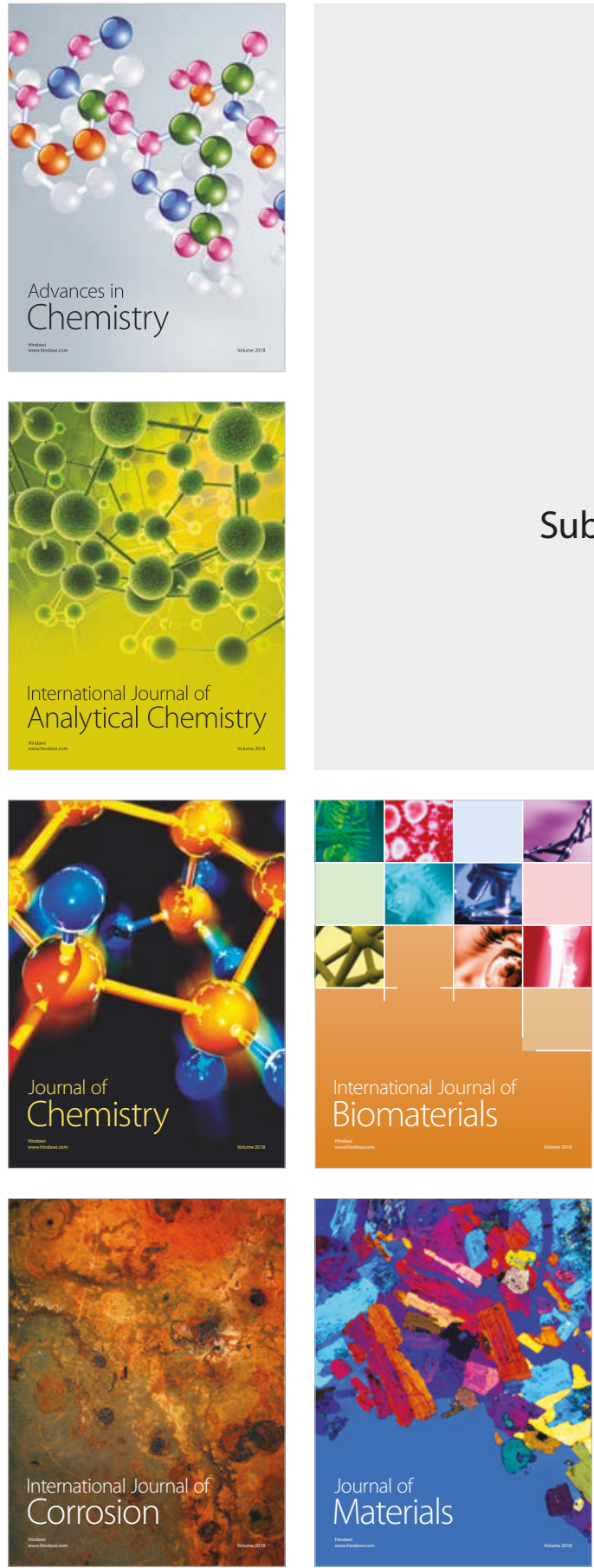

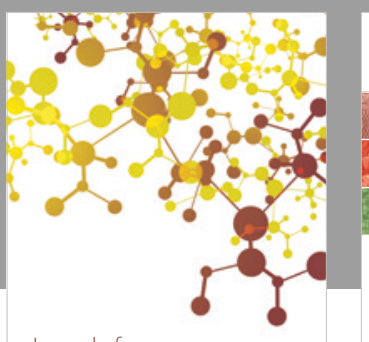

Journal of

Applied Chemistry
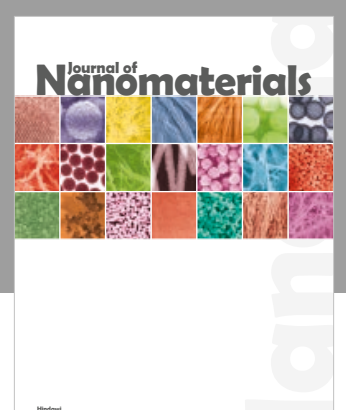

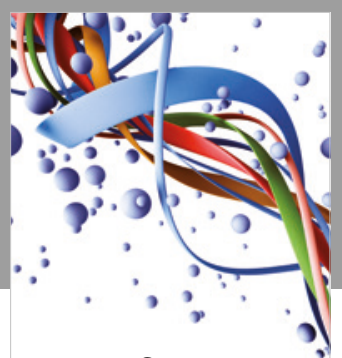

Scientifica

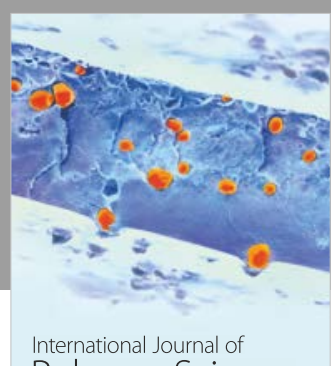

Polymer Science

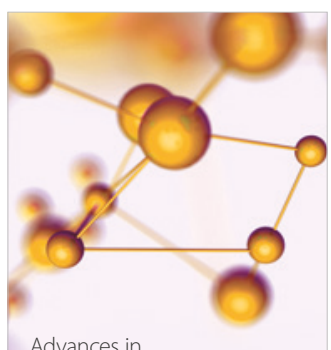

Physical Chemistry
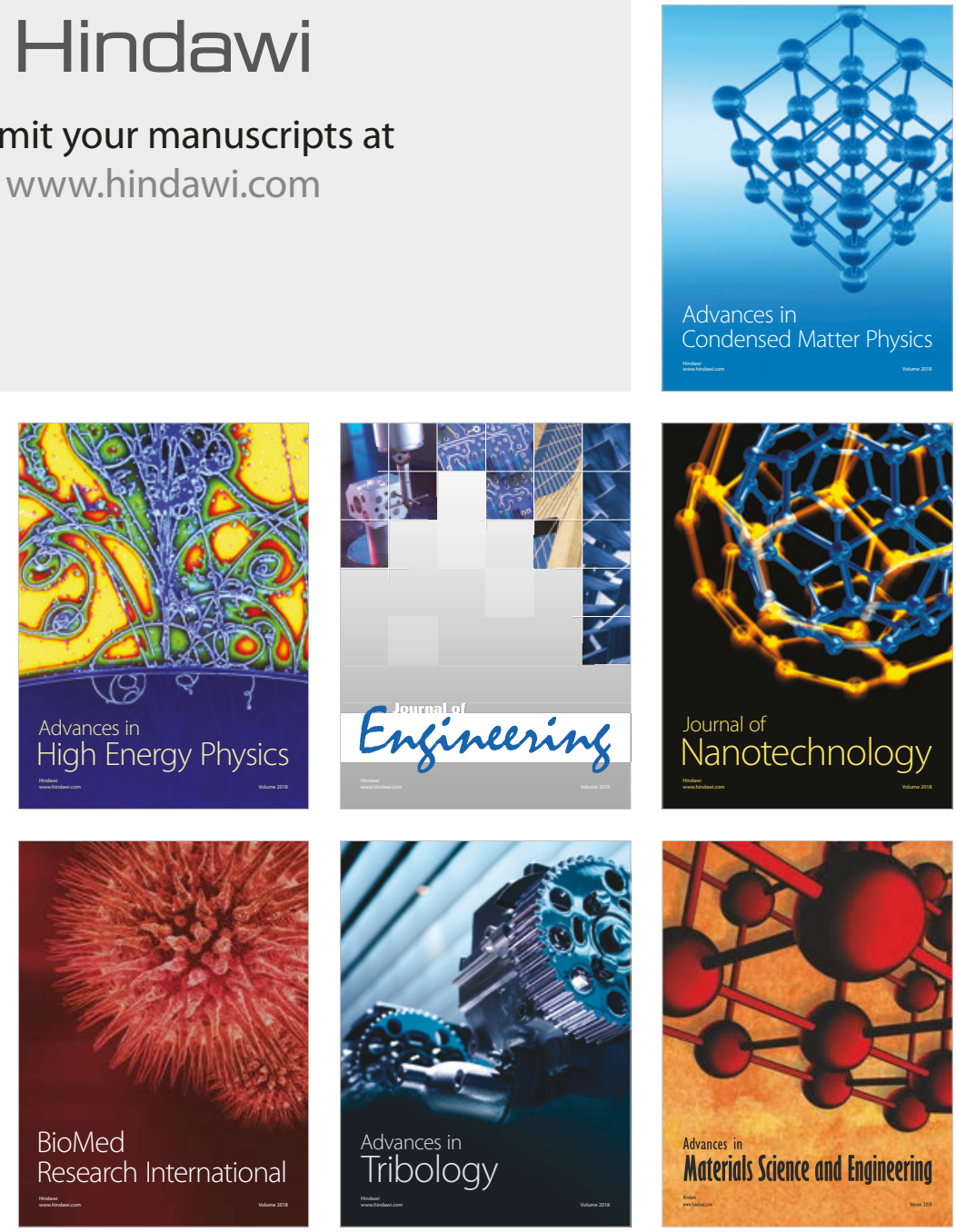